duration at baseline $(\mathrm{R}=0.466, \mathrm{p}=0.009)$. There were no other significant correlations.

Conclusions One year tofacitinib therapy effectively reduced disease activity and systemic inflammation. The therapy also improved functional capacity. JAK inhibition signficantly decreased the number and ratio of $\mathrm{CD} 3+$ and CD4 $+\mathrm{T}$ cells and increased number and ratio of CD19+ B cells in 12 months. Tofacitinib is a safe and effective treatment for rheumatoid arthritis. It makes an effect, partly, via modifing lymphocyte subsets.

Acknowledgements This research was supported by an investigator-initiated research grant from Pfizer.

Disclosure of Interest None declared.

\section{P134/032 SELECTIVE EXPANSION OF A THYMIC-DERIVED AND FUNCTIONALLY COMPETENT REGULATORY T CELL POPULATION BY LOW-DOSE IL-2 THERAPY IN PATIENTS WITH REFRACTORY SLE}

${ }^{1} \mathrm{JY}$ Humrich*, ${ }^{2} \mathrm{C}$ von Spee-Mayer, ${ }^{3} \mathrm{E}$ Siegert, ${ }^{4} \mathrm{M}$ Bertolo, ${ }^{3} \mathrm{~A}$ Rose, ${ }^{3} \mathrm{D}$ Abdirama, ${ }^{4} \mathrm{P}$ Enghard, ${ }^{3} \mathrm{~B}$ Stuhlmüller, ${ }^{3} \mathrm{~F}$ Hiepe, ${ }^{3} \mathrm{~T}$ Alexander, ${ }^{3} \mathrm{E}$ Feist, ${ }^{5} \mathrm{~A}$ Radbruch, ${ }^{3} \mathrm{G}-\mathrm{R}$ Burmester, ${ }^{1} \mathrm{G}$ Riemekasten. ${ }^{1}$ Department of Rheumatology and Clinical Immunology, University Hospital Schleswig-Holstein, Campus Lübeck, Lübeck; ${ }^{2}$ Center for Chronic Immunodeficiency (CCI), University Hospital Freiburg, Freiburg; ${ }^{3}$ Department of Rheumatology and Clinical Immunology; ${ }^{4}$ Department of Nephrology and Intensive Care Medicine, Charité - University Medicine Berlin; ${ }^{5}$ German Rheumatism Research Center (DRFZ) Berlin, Berlin, Germany

\subsection{6/annrheumdis-2018-EWRR2019.121}

Career situation of first and presenting author Instructor.

Objectives An acquired deficiency of interleukin-2 (IL-2) and related defects in regulatory $\mathrm{T}$ cell (Treg) homeostasis play a crucial role in the pathogenesis of SLE. Here, we report the responses of Treg and other lymphocyte subsets to low-dose IL-2 therapy observed during an open-label, uncontrolled, dose adaption, phase $1 / 2 \mathrm{a}$ single-center clinical trial in patients with active and refractory SLE.

Methods 12 patients with active and refractory SLE (SLEDAI $\geq 6$ ) were treated at our site with a low-dose IL-2 regimen consisting of four separate treatment cycles each with daily subcutaneous injections of recombinant human IL-2 (aldesleukin) at single daily doses of either $0.75,1.5$ or 3.0 million IU for five consecutive days. Cells from peripheral blood were analyzed by flow cytometry before and one day after each treatment cycle.

Results All patients showed highly significant cycle- and dosedependent increases in the proportions and absolute numbers of $\mathrm{CD} 3+\mathrm{CD} 4+$ FoxP3 +CD127 lo Treg and of CD25hi expressing cells among Treg. By contrast, we observed no relevant changes in the absolute numbers of $\mathrm{CD} 3+\mathrm{CD} 4+\mathrm{FoxP} 3$ conventional $\mathrm{T}$ cells (Tcon), of $\mathrm{CD} 3+\mathrm{CD} 8+\mathrm{T}$ cells, of $\mathrm{CD} 3$ $+\mathrm{CD} 56+\mathrm{NK} \mathrm{T}$ cells and of CD3-CD56+NK cells. The IL-2 expanded Treg population displayed a preserved suppressive capacity and expressed high levels of the Treg-associated molecules Helios, CD39 and CD137. In addition, we noted robust and dose-dependent increases in the proportions of Treg expressing the proliferation marker Ki67. Although significant increases in the proportions of Ki67 +cells among Tcon and also among other lymphocyte subsets were observed at the end of each treatment cycle, the calculated ratio between Ki67 + Treg and Ki67+ Tcon continuously increased and was significantly higher at the end of the treatment phase, suggesting a preferential targeting of the Treg population.
Conclusions Low-dose IL-2 therapy promotes the selective expansion of a functionally competent and thymic-derived Treg population in patients with refractory SLE. This study also provides novel insights into the pharmacodynamics and the broad biologic effects of low-dose IL-2 therapy.

Disclosure of Interest J. Humrich Consultant for: ILTOO Pharma, C. von Spee-Mayer: None declared, E. Siegert: None declared, M. Bertolo: None declared, A. Rose: None declared, D. Abdirama: None declared, P. Enghard: None declared, B. Stuhlmüller: None declared, F. Hiepe: None declared, T. Alexander: None declared, E. Feist: None declared, A. Radbruch: None declared, G.-R. Burmester: None declared, G. Riemekasten Consultant for: ILTOO Pharma.

\section{P136 THE EFFECTS OF LY294002, THE PHOSPHOINOSITIDE 3-KINASE INHIBITOR, ON NEUTROPHIL APOPTOSIS IN PATIENTS WITH RHEUMATOID ARTHRITIS AND COLLAGEN-INDUCED ARTHRITIS IN MICE}

X Huang, T Li*, S Zheng, W Deng, Y Huang, Z Huang, Q Huang, Z Huang. Department of Rheumatology and Immunology, Guangdong Second Provincial General Hospital, Guangzhou, China

\subsection{6/annrheumdis-2018-EWRR2019.122}

Career situation of first and presenting author Young investigator.

Introduction Neutrophils act as a first line in host defense, they also play important roles on acute and chronic inflammation. Rheumatoid arthritis (RA) is a chronic inflammatory autoimmune disease characterized by persistent joint inflammation. ${ }^{1}$ Previous studies have shown that delayed apoptosis of neutrophils correlated with the pathogenesis of RA. ${ }^{2}$

Objectives The aim of this study was to investigate the effects of LY294002, the phosphoinositide 3-kinase(PI3K) inhibitor, on neutrophil apoptosis in RA patients and collagen-induced arthritis (CIA) in mice.

Methods The peripheral blood from untreated RA patients who satisfy the 1987 ACR classification criteria for RA $(n=20)$ and healthy volunteers $(H C)(n=10)$ were used to isolate neutrophils by density gradient centrifugation. The neutrophils were cultured with or without LY294002 in vitro for 18 hour. The rate of neutrophils apoptosis were analyzed by flow cytometry. The intracellular protein levels of PI3K-Akt pathway in neutrophils were measured by Western blot. CIA mice were treated with vehicle or LY294002, and the incidence, arthritis score, histological assessment as well as the apoptosis rate of neutrophils from peripheral blood and marrow were analyzed.

Results The peripheral blood neutrophils apoptosis delayed in RA patients as compared with neutrophils from HC. As compare with the $\mathrm{HC}$, the expression of phosphorylation $\mathrm{PI} 3 \mathrm{~K}(\mathrm{p}-$ PI3K) and p-Akt were increased in RA patients. LY294002 could significantly promote neutrophils apoptosis in peripheral blood from RA patients. LY294002 blocked the activation of PI3K-Akt and inhibited the expression of p-Akt. Moreover, LY294002 treatment delayed the onset, reduced the severity of arthritis in CIA mice. We also found that neutrophils from both peripheral blood and bone marrow delayed apoptosis in CIA mice as compared with the control group, and LY294002 treatment facilitated the neutrophil apoptosis from peripheral blood and marrow in CIA mice.

Conclusions This data suggested that delayed apoptosis of neutrophils, which might through the activation of PI3K-Akt 
pathway, played an important role in the development of RA. LY294002 could delay the onset and reduce the severity of arthritis in CIA mice through the promotion of neutrophils apoptosis. It might open a new door to the future clinical treatment of RA.

\section{REFERENCES}

1. Mclnnes IB, Schett $G$. The pathogenesis of rheumatoid arthritis. N Engl J Med 2011;365(23):2205-19.

2. Wright HL, Moots RJ, Edwards SW. The multifactorial role of neutrophils in rheumatoid arthritis. Nat Rev Rheumatol 2014;10(10):593-601.

Acknowledgements This work was supported by Youth Science Foundation of Guangdong Second Provincial General Hospital (No. YQ2016-002).

Disclosure of Interest None declared.

\section{P138/010 INVESTIGATING GENE EXPRESSION PATTERNS AND FUNCTION OF TOLEROGENIC DENDRITIC CELLS}

L Nwosu*, K Smith, C Hilkens, J Isaacs. Institute of Cellular Medicine, Newcastle University, Newcastle upon Tyne, UK

\subsection{6/annrheumdis-2018-EWRR2019.123}

Career situation of first and presenting author Post-doctoral fellow.

Introduction Dendritic cells (DCs) are professional antigen-presenting cells and play a major role in immune system responses and function. They express a repertoire of toll-like receptors (TLRs) that recognise pathogens. Tolerogenic dendritic cell (tolDC) therapy is a promising cellular therapy that aims to restore tolerance in autoimmune disease. Exposure to dexamethasone leads to the expression of TLR2; however, expression of this marker is not homogeneous. Some tolDCs have low TLR2 expression while some others have high TLR2 expression. Despite these differences in TLR2 expression, we hypothesised that tolDCs are homogenous in terms of their gene expression and function.

Objectives The study aimed to investigate the gene expression and function of tolDCs to determine if expression patterns and function seen in low TLR2 expressing tolDCs are similar to those of high TLR2 expressing tolDCs.

Methods Peripheral blood CD14 ${ }^{+}$monocytes, obtained from healthy individuals $(n=8)$ were differentiated for 7 days into tolDCs using IL-4, granulocyte-macrophage colony-stimulating factor (GM-CSF), dexamethasone and Vitamin D3. As a control, mature DCs were differentiated using IL-4, GM-CSF and IFN- $\gamma$. TolDCs were stained for TLR2 and fluorescence activated cell sorted (FACS) as the 20\% lowest TLR2 expressing tolDCs and the 20\% highest TLR2 expressing tolDCs. TolDC phenotype was explored using flow cytometry, gene expression of pro-inflammatory, anti-inflammatory and migratory genes by quantitative PCR and CD40-Ligand re-stimulation and mixed lymphocyte reaction assay to investigate function.

Results Phenotypic marker expression was different between tolDCs and mature DCs, with tolDCs expressing low levels of CD83 and CD86 and high levels of HLA-DR, latency-associated peptide (LAP) and TLR2. In general, TLR2Low and TLR2High tolDCs showed similar phenotypic properties, gene expression patterns and tolerogenic functions. TLR2High tolDCs had increased LAP and HLA-DR expression. TLR2Low tolDCs had higher gene expression for CCR7 and TNF- $\alpha$. Cytokine (IL-6 and IL-10) production for both TLR2Low and
TLR2High tolDCs was not significantly different upon re-stimulation with CD40-ligand and both populations had similar immunosuppressive capacity for $\mathrm{CD} 4+\mathrm{T}$ cells compared to mature DCs.

Conclusions Both populations of tolDCs displayed similar gene expression profiles and phenotypic properties for the majority of characteristics investigated. The minor differences observed may be attributable to stochastic differences in dendritic cell exposure to, or uptake of dexamethasone. Despite these differences, all tolDCs function similarly, which is the most important factor when considering tolDCs as a therapy.

Disclosure of Interest None declared.

\section{P140 IDENTIFICATION OF AFFIMERS THAT BIND TO THE IL- 7R AND INHIBITS THE IL-7 SIGNALLING CASCADE}

${ }^{1} \mathrm{D}$ Perez-Witzke, ${ }^{2} \mathrm{C}$ Tiede, ${ }^{3} \mathrm{I}$ Robinson, ${ }^{2} \mathrm{D}$ Tomlinson, ${ }^{1} \mathrm{P}$ Emery, ${ }^{1} \mathrm{~F}$ Ponchel ${ }^{*}$. ${ }^{1}$ Leeds Institute of Rheumatic and Musculoskeletal Medicine; ${ }^{2}$ BioScreening Technology group; ${ }^{3}$ Leeds Institute of Cardiovascular and Metabolic Medicine, University of Leeds, Leeds, UK

\subsection{6/annrheumdis-2018-EWRR2019.124}

Career situation of first and presenting author Student for a master or a $\mathrm{PhD}$.

Introduction IL-7R is a heterodimer constituted by the IL-7R alpha $(\alpha)$ chain (CD127) and the common gamma (g) chain (CD132). IL-7 binding to IL-7R expressed on CD4+ T cells induces a survival signal. The IL-7/IL-7R signalling axis has been validated as a therapeutic target for treatment of both Tcell driven autoimmune diseases (AIDs) ${ }^{1}$ and $\mathrm{T}$ Acute Lymphoblastic Leukaemia (T-ALL). ${ }^{2}$ Affimers are small and stable artificial proteins which bind with nanomolar affinities to human proteins and can block protein-protein interactions. ${ }^{3}$ They are becoming widespread owing to their stability, ease of production and versatility.

Objectives Identify Affimers that recognise the IL-7R $\alpha$ and inhibits the IL-7 signalling cascade. This may result in an attractive approach for the treatment of both T-cell driven autoimmune diseases and T-ALL.

Methods The type-II Affimer library $\left(10^{10}\right)$ was interrogated by Phage display using fully glycosylated human IL-7R $\alpha$ ectodomain (ECD). PhageELISA and DNA sequencing were used to either obtain or elucidate the unique binders (Affimers), respectively. Affimers were produced as His-Tagged proteins $(\sim 13 \mathrm{kDa})$ in E. coli and purified using IMAC. Affimer binding to IL-7R $\alpha$ was confirmed by pull-down assays (soluble) and Flow cytometry (membrane). An IL-7 reporter assay using HEK-IL7R (HEK293 cells stably transfected with the IL-7R) was developed and the biological effect of the Affimers was elucidated.

Results We have screened an Affimer library using human ECD-IL7R $\alpha$ and after three consecutive panning rounds, 20 Affimers were raised as shown by PhageELISA and DNA sequencing. From these, 17 were able to pull-down the soluble ECD-IL7R $\alpha$ and 7 stained specifically HEK-IL7R cells (by flow cytometry using anti-His Tag Abs). Finally, we have identified 3 Affimers (1, 42 and 96) that showed inhibition of the IL-7 signalling cascade on HEK-IL7R cells.

Conclusions Our work demonstrates the possibility of screening an Affimer library for a cytokine-receptor target, and selecting specific binders, some of which showed the desired antagonist activity of the cytokine signalling cascade. IL-7 itself is a validated target, so this work offers an alternative to antibody-mediated protein interference. With further biological 